\title{
Quantitative Reconstruction and Analysis of HRTEM Complex Exit Waves using Inline Holography
}

\author{
Colin Ophus, ${ }^{1}$ Jim Ciston ${ }^{1}$, Peter Ercius ${ }^{1}$ and Christoph Koch $^{2}$ \\ ${ }^{1}$ National Center for Electron Microscopy, Lawrence Berkeley National Laboratory, CA, USA \\ ${ }^{2}$ Institut für Experimentelle Physik, Universität Ulm, Ulm, Germany
}

In high resolution transmission electron microscopy (HRTEM), we record the intensity of an electron plane wave after it has interacted with an electron-transparent sample. This interaction between fast electrons and the positive electrostatic potential of the sample is encoded upon the phase of the electron plane wave, which is not directly measurable in experiments. However, after the electron- sample interaction, Fresnel diffraction of the wave transfers some of the information into the plane wave intensity. By controlling the focal distance of the objective electron lens, we can also introduce coherent wave aberrations which further modulate the hidden phase information. After capturing a series of measured intensity micrographs with varying defocus values, there are several phasing algorithms we can apply to recover the full complex exit wave. The most common of these methods is the well-known Gerchberg-Saxton algorithm [1,2].

The Gerchberg-Saxton algorithm is fast, simple to implement, can handle both coherent wave aberrations and incoherence effects (limited spatial and temporal coherence), and is robust in the presence of noise. However, in a previous study we showed that the convergence of this algorithm depends strongly on the spatial frequency $q_{0}$. Specifically, the characteristic number of iterations $N$ required for convergence is approximately $N \sim 0.3 \lambda^{-2} F^{-2} q_{0}{ }^{-4}$ [3], where $\lambda$ is the electron wavelength and $F$ is the maximum defocus value. As the spatial frequency $q_{0}$ is lowered (longer distances in the real-space exit wave), the number of iterations required to recover it increase to the fourth power. For example, recovering all spatial frequencies of the full exit wave over a field of view of $20 \mathrm{~nm}\left(q_{0}=0.05\right.$ $\left.\mathrm{nm}^{-1}\right)$, in a $300 \mathrm{kV}$ HRTEM experiment $(\lambda=0.00197 \mathrm{~nm})$ with a maximum defocus $F=20 \mathrm{~nm}$ would require over 30 million iterations, far more than a rough practical limit of 1000 iterations. The low spatial frequencies are especially important in HRTEM sample geometry studies, because the sample thickness is encoded there [3]. Figure 1 demonstrates the information that is lost when the low spatial frequencies are attenuated or missing from a complex exit wave.

In this study, we introduce a new exit wave reconstruction algorithm that dramatically speeds convergence, building on our previous work [3,4]. We use Gerchberg-Saxton iterations to recover the medium and high spatial frequencies, and a modified transport of intensity equation to solve the low spatial frequencies, similar to the composite approach proposed by Gureyev [5]. We use simulated focal series to test the efficacy of our new algorithm. We also discuss the effects of noise and open boundary conditions on the results. Finally we apply the algorithm to experimental datasets (wedge, nanoparticles, etc) and quantitatively access the results. Figure 2 shows an experimental focal series reconstructed using our method [6].

\section{References:}

[1] RW Gerchberg and WO Saxton. A practical algorithm for the determination of phase from image and diffraction plane pictures. Optik, 35 (1972) 237, 
[2] W.K. Hsieh, FR Chen, J.J. Kai, and A.I. Kirkland. Resolution extension and exit wave reconstruction in complex HREM. Ultramicroscopy, 98 (2004) 99-114,

[3] C. Ophus and T. Ewalds. Guidelines for quantitative reconstruction of complex exit waves in HRTEM, Ultramicroscopy, 113 (2012) 88-95

[4] CT Koch. Full-resolution inline electron holography (FRIH). In European Microscopy Congress EMC2012 in Manchester, UK, volume 139, 2012.

[5] TE Gureyev. Composite techniques for phase retrieval in the Fresnel region. Optics communications, 220 (2003) 49-58

[6] This work was performed at the National Center for Electron Microscopy, which is supported by the Office of Basic Energy Sciences of the U.S. Department of Energy under Contract No. DEAC0205CH11231. We thank Alain Thorel for providing the Si3N4 sample.
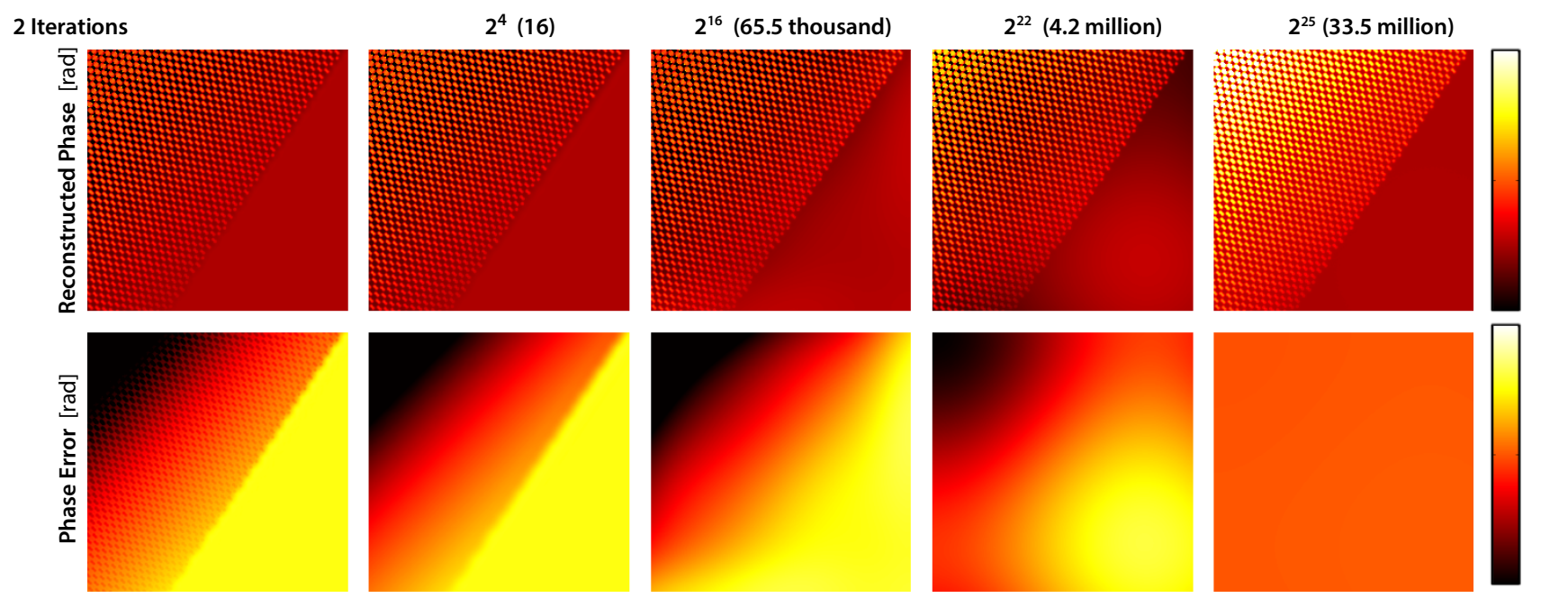

Figure 1: (top) Reconstructed phase on subset of simulation for various numbers of iterations. (bottom) Difference image between reconstructed and simulated exit wave phase. Note that the atomic structure (high spatial frequencies) is recovered within $\approx 16$ iterations. However, completely reconstructing the phase wedge due to the thickness variation requires over 30 million iterations.

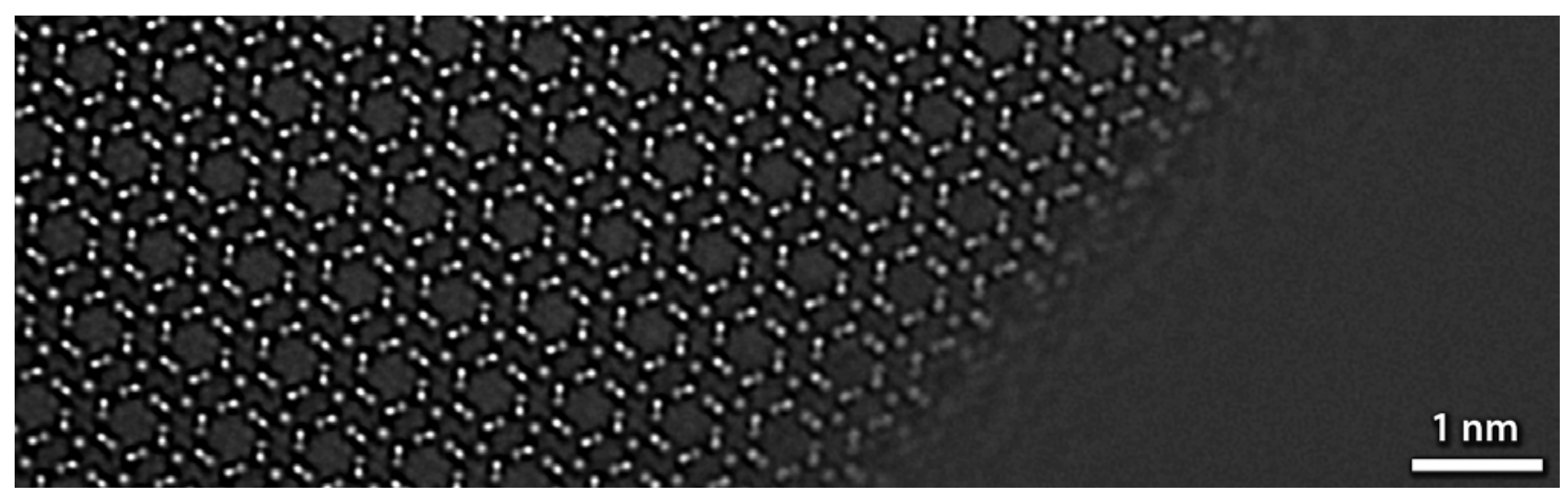

Figure 2: Exit wave intensity reconstructed from an experimental focal series of a $\beta-\mathrm{Si}_{3} \mathrm{~N}_{4}$ sample. 